\title{
A short remark on a new Fibonacci-type sequence
}

\author{
Krassimir T. Atanassov ${ }^{1,2}$ \\ ${ }^{1}$ Department of Bioinformatics and Mathematical Modelling \\ IBPhBME - Bulgarian Academy of Sciences, \\ Acad. G. Bonchev Str. Bl. 105, Sofia-1113, Bulgaria \\ e-mail: krat@bas.bg \\ ${ }^{2}$ Intelligent Systems Laboratory \\ Prof. Dr Asen Zlatarov University, Burgas-8010, Bulgaria
}

Received: 1 January 2021

Accepted: 4 May 2021

Abstract: A new Fibonacci-type sequence is constructed and for it is proved that it has a basis with 24 elements.

Keywords: Arithmetic function, Fibonacci sequence.

2020 Mathematics Subject Classification: 11B39.

\section{Introduction}

In this short remark, we describe a sequence generated by two arbitrary one-digit natural numbers and the arithmetic function $\psi$, defined and studied in [1,2].

Everywhere we use the natural number $n$ of the following form

$$
n=\sum_{i=1}^{k} a_{i} \cdot 10^{k-i} \equiv \overline{a_{1} a_{2} \ldots a_{k}}
$$

where $a_{i}$ is a natural number and $0 \leq a_{i} \leq 9(1 \leq i \leq k)$.

Following $[1,2]$, we define a function denoted by $\varphi$ by:

$$
\varphi(n)= \begin{cases}0, & \text { if } n=0, \\ \sum_{i=1}^{k} a_{i}, & \text { if } n>0 .\end{cases}
$$


Now, we define a sequence of functions $\varphi_{0}, \varphi_{1}, \varphi_{2}, \ldots$, where for each natural number $l$ :

$$
\begin{aligned}
\varphi_{0}(n) & =n, \\
\varphi_{l+1} & =\varphi\left(\varphi_{l}(n)\right) .
\end{aligned}
$$

Obviously, for every $l \in \mathbb{N}, \varphi_{l}: \mathbb{N} \rightarrow \mathbb{N}$. Since for $k>1$

$$
\varphi(n)=\sum_{i=1}^{k} a_{i}<\sum_{i=1}^{k} a_{i} \cdot 10^{k-i}=n,
$$

then for every $n \in \mathbb{N}$ there exists $l \in \mathbb{N}$ such that

$$
\varphi_{l}(n)=\varphi_{l+1}(n) \in \Delta \equiv\{0,1,2, \ldots, 9\} .
$$

Following [1,2], let the function $\psi$ be defined by

$$
\psi(n)=\varphi_{l}(n),
$$

where

$$
\varphi_{l+1}(n)=\varphi_{l}(n)
$$

Hence, $\psi: \mathbb{N} \rightarrow \Delta$.

The following equalities are proved in $[1,2]$ for every two natural numbers $m$ and $n$ :

$$
\begin{aligned}
\psi(0) & =0 \\
\psi(m+n) & =\psi(\psi(m)+\psi(n)), \\
\psi(n+9) & =\psi(n) .
\end{aligned}
$$

\section{Main result}

Now, having in mind that

$$
\psi(10 m+n)=\psi(m+n)
$$

(see $[1,2])$, we obtain sequentially for two arbitrary natural numbers $a, b \in\{0,1, \ldots, 9\}$ :

\begin{tabular}{|l||l|}
\hline \hline 1 & $a$ \\
2 & $b$ \\
3 & $\psi(\overline{b a})=\psi(10 b+a)=\psi(a+b)$ \\
4 & $\psi(b+\psi(a+b))=\psi(a+2 b)$ \\
5 & $\psi(\psi(a+b)+\psi(a+2 b))=\psi(2 a+3 b)$ \\
6 & $\psi(\psi(a+2 b)+\psi(2 a+3 b))=\psi(3 a+5 b)$ \\
7 & $\psi(5 a+8 b)$ \\
8 & $\psi(8 a+13 b)=\psi(8 a+4 b)$ \\
9 & $\psi(13 a+12 b)=\psi(4 a+3 b)$ \\
10 & $\psi(3 a+7 b)$ \\
11 & $\psi(7 a+b)$ \\
12 & $\psi(a+8 b)$ \\
13 & $\psi(8 a+9 b)=\psi(8 a)$ \\
\hline
\end{tabular}

Cont'd 


\begin{tabular}{|l||l|}
\hline 14 & $\psi(9 a+8 b)=\psi(8 b)$ \\
15 & $\psi(8 a+8 b)$ \\
16 & $\psi(8 a+7 b)$ \\
17 & $\psi(7 a+6 b)$ \\
18 & $\psi(6 a+4 b)$ \\
19 & $\psi(4 a+b)$ \\
20 & $\psi(a+5 b)$ \\
21 & $\psi(5 a+6 b)$ \\
22 & $\psi(6 a+2 b)$ \\
23 & $\psi(2 a+8 b)$ \\
24 & $\psi(8 a+b)$ \\
25 & $\psi(a+9 b)=\psi(a)=a$ \\
26 & $\psi(9 a+b)=\psi(b)=b$. \\
\hline \hline
\end{tabular}

Therefore, this sequence has the form

$$
\begin{aligned}
\alpha_{0} & =a, \\
\alpha_{1} & =b, \\
\alpha_{n+2} & =\psi\left(10 \alpha_{n+1}+\alpha_{n}\right), \text { for } n \geq 0 .
\end{aligned}
$$

Let the sequence of natural numbers $a_{1}, a_{2}, \ldots$ be given and let

$$
c_{i}=\psi\left(a_{i}\right)(i=1,2, \ldots) .
$$

Hence, following $[1,2]$, we deduce the sequence $c_{1}, c_{2}, \ldots$ from the former sequence. If $k$ and $l$ exist such that $l \geq 0$,

$$
c_{i+l}=c_{k+i+l}=c_{2 k+i+l}=\cdots
$$

for $1 \leq i \leq k$, then we will say that

$$
\left[c_{l+1}, c_{l+2}, \ldots, c_{l+k}\right]
$$

is the base of the sequence $a_{1}, a_{2}, \ldots$ of length $k$ and with respect to function $\psi$.

Therefore, the following assertion is valid.

Theorem. The sequence

$$
a, b, \psi(10 b+a), \psi(10 \psi(a+b)+b), \ldots
$$

has a basis of length 24 .

Analogously, we can construct the other possible sequence

$$
a, b, \psi(10 a+b), \psi(10 \psi(a+b)+a), \ldots
$$

with $(n+2)$-nd term

$$
\alpha_{n+2}=\psi\left(\alpha_{n+1}+10 \alpha_{n}\right), \text { for } n \geq 0,
$$

where $\alpha_{0}=a, \alpha_{1}=b$. For it, the Theorem will be valid, again. 


\section{Conclusion}

A new type of Fibonacci like sequences has been introduced. In future, the standard Fibonacciform of these sequences will be extended to a Tribonacci, and more generally, $k$-bonacci-forms.

\section{References}

[1] Atanassov, K. (1985). An arithmetical function and some of its applications. Bulletin of Number Theory and Related Topics, IX(1), 18-27.

[2] Atanassov, K. (2015). A digital arithmetical function and some of its applications. Proceedings of the Jangjeon Mathematical Society, 18(4), 511-528. 